Voix et Images

volxetimages

\title{
L’Orient dans les récits des voyageurs québécois de la seconde moitié du vingtième siècle
} Une expérience de déperdition de soi

\section{The Orient in the Narratives of Quebec Travellers in the Second} Half of the Twentieth Century An Experience of Loss of Self

\section{Pierre Rajotte}

Volume 31, numéro 1 (91), automne 2005

Figures et contre-figures de l'orientalisme

URI : https://id.erudit.org/iderudit/011922ar

DOI : https://doi.org/10.7202/011922ar

Aller au sommaire du numéro

\section{Éditeur(s)}

Université du Québec à Montréal

\section{ISSN}

0318-9201 (imprimé)

1705-933X (numérique)

\section{Découvrir la revue}

Citer cet article

Rajotte, P. (2005). L’Orient dans les récits des voyageurs québécois de la seconde moitié du vingtième siècle : une expérience de déperdition de soi. Voix et Images, 31(1), 15-31. https://doi.org/10.7202/011922ar
Résumé de l'article

Au cours de la seconde moitié du vingtième siècle, les récits des voyageurs québécois en Orient sont marqués par une nouvelle dynamique relationnelle entre le soi et l'autre. Le désir de retrouver les traces de sa propre culture dans celle de l'autre laisse progressivement place à une expérience initiatique de déperdition de soi et de ses repères. En témoignent le choix des destinations (l'Extrême-Orient plutôt que la Terre sainte), la remise en question des représentations ethnocentrées, constitutives du discours nationaliste, l'intérêt marqué pour le présent et l'avenir des civilisations orientales et non plus uniquement pour leur passé mythique, mais surtout la perception d'une altérité inversée, provoquée notamment par le regard de l'autre, qui révèle aux voyageurs leur propre étrangeté. 


\section{L'ORIENT DANS LES RÉCITS \\ DES VOYAGEURS QUÉBÉCOIS}

DE LA SECONDE MOITIÉ DU VINGTIËME SIÉCLE.

Une expérience de déperdition de soi

$+++$

PIERRE RAJOTTE

Université de Sherbrooke

\section{RÉSUMÉ}

Au cours de la seconde moitié du vingtième siècle, les récits des voyageurs québécois en Orient sont marqués par une nouvelle dynamique relationnelle entre le soi et l'autre. Le désir de retrouver les traces de sa propre culture dans celle de l'autre laisse progressivement place à une expérience initiatique de déperdition de soi et de ses repères. En témoignent le choix des destinations (l'Extrême-Orient plutôt que la Terre sainte), la remise en question des représentations ethnocentrées, constitutives du discours nationaliste, l'intérêt marqué pour le présent et l'avenir des civilisations orientales et non plus uniquement pour leur passé mythique, mais surtout la perception d'une altérité inversée, provoquée notamment par le regard de l'autre, qui révèle aux voyageurs leur propre étrangeté. 
Le dépaysement, comme celui que m'impose l'Orient,

prescrit une forme d'effacement temporaire,

qui est en fait un dessaisissement salutaire.

Car on ne se fréquente jamais d'aussi près que lorsqu'on s'éloigne,

qu'on se détourne de soi,

j'entends de ce soi appris et apprivoisé, affiché, monté en épingle ${ }^{1}$.

Dans les récits de voyage québécois du dix-neuvième siècle et du début du vingtième siècle, l'Orient ne présente généralement d'intérêt que dans la mesure où il permet aux voyageurs de se réapproprier un patrimoine culturel et religieux ${ }^{2}$. Dans le contexte éminemment nationaliste de l'époque, le pèlerinage oriental a pour fonction non pas de déstabiliser mais de renforcer une identité canadienne, à la fois catholique et française. Aussi, la réaction la plus courante des voyageurs consistet-elle à se servir de la culture de l'autre pour conforter la leur et, plus précisément, à rechercher les traces de leur propre culture dans celle de l'autre. Encore au début du vingtième siècle, plusieurs voyageurs ne ratent aucune occasion de célébrer le Canada et le catholicisme par pays interposés. Au sujet d'un marché arabe d'Alger, par exemple, l'abbé François-Xavier-Adolphe Dulac écrit: "Que de guenilles, que de saletés; c'est un pandémonium indescriptible! Pour nous, Canadiens, nous sommes contents d'avoir vu semblable spectacle, et cela nous prouve, de toute évidence, que nos pauvres au Canada, sont des riches à côté des pauvres d'Alger ${ }^{3}$.»

Au cours de la seconde moitié du vingtième siècle toutefois, cette approche ethnocentrique laisse peu à peu place au désir de la fuite, de l'évasion et du détachement à l'égard de sa propre culture. Comme le mentionne Maurice Lebel dans son récit de voyage en Turquie en 1957, l'un des plus grands plaisirs du voyageur devient «le dépaysement, le changement d'optique, l'art de se laisser prendre par des spectacles nouveaux, la chaude sympathie pour des mondes différents du sien propre ${ }^{4}$ ». En fait, plus on avance dans le vingtième siècle, plus on voyage pour renoncer à ce que l'on est et pour se libérer «de mille liens et attaches que l'on croyait indispensables à son bien-être ${ }^{5}$ ». André Belleau estime même en 1986, dans son bref récit «Maroc sans noms propres», que le voyage exige «que l'on tente de sortir de soi ${ }^{6} »$.

$$
+++
$$

1 André Carpentier, Mendiant de l'infini. Fragments nomades, Montréal, Boréal, 2002, p. 54. Désormais, les références à ce récit seront indiquées par le sigle $M I$, suivi du folio, et placées entre parenthèses dans le texte. 2 Voir Pierre Rajotte, «The Self and the Other: Writings of Quebec Travellers in the Middle East at the End of the 19th Century ", Canadian Literature, nº 174, 2002, p. 98-115; Id., "La représentation de l'Autre dans les récits de voyage en Terre sainte à la fin du XIX siècle", Études françaises, vol. 32, n 3, 1996, p. 94-113; voir également Pierre Rajotte, avec la collaboration d'Anne-Marie Carle et de François Couture, Le récit de voyage au XIXe siècle. Aux frontières du littéraire, Montréal, Triptyque, 1997. 3 L'abbé F.-X.-A. Dulac, Aux pays de Jésus, Québec, Imprimerie «Le Soleil», 1922, p. 70. 4 Maurice Lebel, Images de la Turquie, Québec, Librairie de l'Action catholique, 1957, p. 3. 5 Michel Lemieux, Voyage au Levant, Sillery, Septentrion, 1992, p. 75. Désormais, les références à ce récit seront indiquées par le sigle $V L$, suivi du folio, et placées entre parenthèses dans le texte. 6 André Belleau, « Maroc sans noms propres », Surprendre les voix, Montréal, Boréal, 1986, p. 55. 
On assiste donc peu à peu à la mise en place d'un véritable «rituel de déperdition de soi et de ses repères ${ }^{7}$ ", pour reprendre l'expression de Rachid Amirou. Différents facteurs l'illustrent bien, comme l'attrait suscité par de nouvelles destinations extrêmes-orientales, la remise en question des représentations traditionnelles, constitutives du discours nationaliste, l'intérêt marqué pour le présent et l'avenir de la civilisation orientale (et non plus seulement pour son passé mythique), mais surtout la perception d'une altérité inversée, provoquée notamment par le regard de l'autre qui révèle aux voyageurs leur propre étrangeté.

\section{LOIN DU PAYS, LOIN DE SOI}

D'entrée de jeu, en parcourant un corpus de 71 récits de voyage en Orient publiés dans la seconde moitié du vingtième siècle, on constate que l'intérêt des voyageurs n'est plus circonscrit à la Terre sainte comme au dix-neuvième siècle. En excluant les récits de voyage autour du monde ${ }^{8}$, mes recherches m'ont permis jusqu'à présent de retrouver 20 récits publiés en volumes qui portent spécifiquement sur le Proche et le Moyen-Orient et 51 qui traitent d'une région ou d'une autre de l'Extrême-Orient.

\section{Production des récits de voyage en Orient (vingtième siècle)}

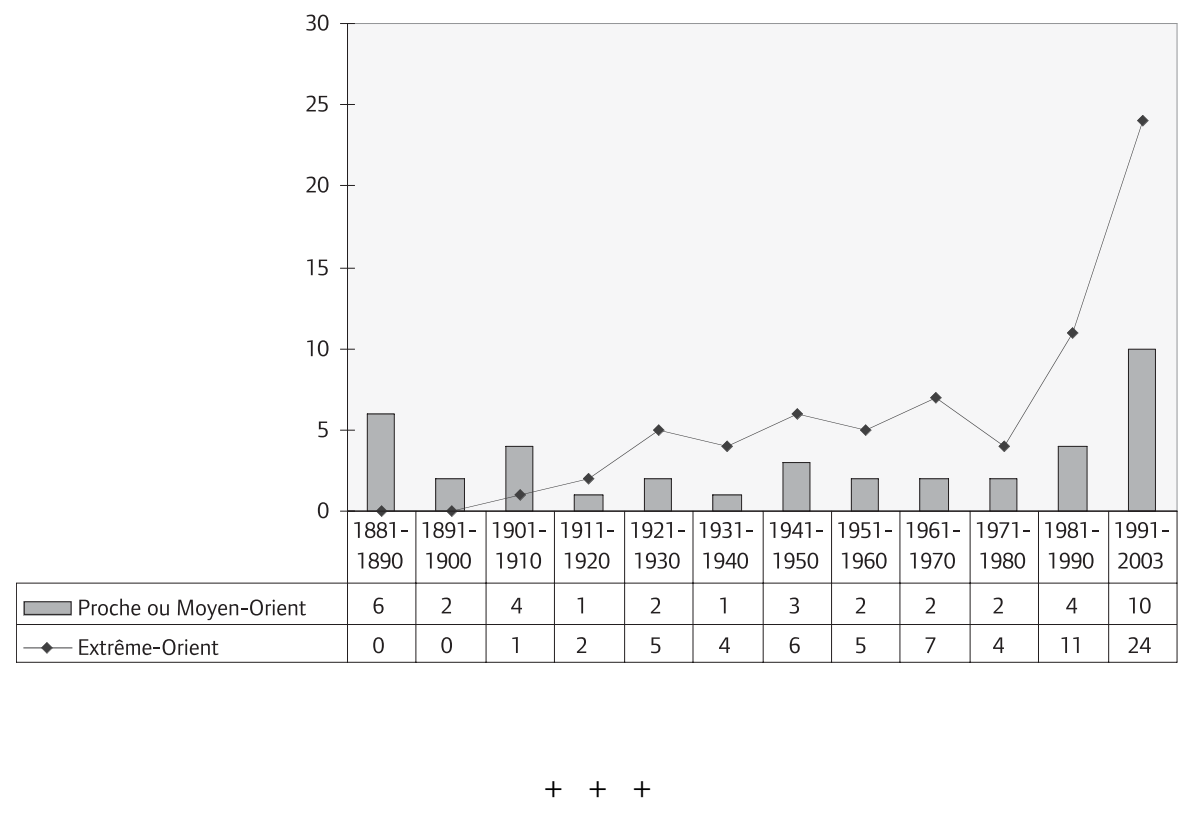

7 Rachid Amirou, Imaginaire touristique et sociabilités du voyage, Paris, Presses universitaires de France, 1995 , p. 132. 8 Pour le tableau ci-joint, nous n'avons pas retenu les récits de voyage autour du monde, ni les recueils de récits de voyage dans diverses régions du monde qui traitent bien souvent du Proche, du Moyen et de l'Extrême-Orient sans être entièrement consacrés à ces destinations. 
Certes, le progrès des transports et le développement des circuits touristiques rendent maintenant plus accessibles les multiples régions de l'Orient, mais l'inconnu recherché par les voyageurs n'est pas étranger à la fascination qu'ils éprouvent pour certains pays d'Asie, comme le Japon, la Chine et l'Inde, qui passent pour avoir les formes de civilisations les plus éloignées de la nôtre. Le dépaysement qu'imposent ces destinations offre au voyageur l'occasion d'apprendre à regarder avec un œil nouveau, l'occasion d'« ébranler toute sa personne, jusqu'à l'étrangeté» (MI, 230), de «se déposséder de soi en milieu dépossédé de tout» (MI, 171), comme l'estime André Carpentier dans son récit de voyage au Tibet. Autrement dit, parcourir ces pays exotiques favorise un décentrement identitaire et une forme d'opposition au récit de voyage conçu comme dépositaire d'une culture et d'une tradition que l'écriture doit galvaniser. À la limite, l'identité du soi et l'identité du même peuvent cesser de se recouvrir, voire se dissocier entièrement, «mettant en quelque sorte à nu l'ipséité du soi sans le support de la mêmeté ${ }^{9}$ ", pour reprendre une formulation de Paul Ricœur.

Cette rupture volontaire avec des appartenances premières entraine des modifications au sein de la structure des récits de voyage. Les voyageurs de la première moitié du siècle associaient presque systématiquement le début de leur récit au regret de quitter leur pays, leur ville, leur foyer, pour se faire pardonner leur voyage à l'étranger: "Au revoir, tours crénelées de Notre-Dame, flèches altières de SaintJacques, de Saint-Pierre, de Sainte-Cunégonde et de Saint-Henri; étincelantes coupoles de Saint-Jean-Baptiste et de la cathédrale. C'est lorsqu'on se sépare des bonnes choses qu'on en connaît mieux le prix ${ }^{10}$ », écrivait Émile Miller en 1923. Or, les récits de la seconde moitié du siècle escamotent souvent ce rituel de séparation. La plupart des auteurs se lancent d'entrée de jeu dans l'ailleurs, comme si quitter leur pays n'impliquait aucun regret ni aucune émotion particulière. De même, leur retour n'est plus que très rarement l'occasion de célébrer leur lieu d'origine, comme c'était le cas auparavant. Souvent réduit à sa plus simple expression dans les récits, le retour met fin au rêve du voyageur qui a l'impression d'être "rentré mais toujours là-bas» $(M I, 232)$ et qui se rabat mélancoliquement sur ses souvenirs, voire sur l'espoir de bientôt repartir.

\section{SE DÉSALIÉNER D’UNE PERCEPTION ETHNOCENTRÉE}

Le dépouillement de soi se reflète également dans la remise en question des idées reçues et des représentations axiologiques héritées des discours nationalistes et coloniaux. Ainsi, dans leur récit de voyage intitulé Deux innocents en Chine rouge, publié en 1961, Jacques Hébert et Pierre E. Trudeau annoncent leur «intention de corriger la notion du Péril jaune ${ }^{11}$ » et, partant, de se libérer des peurs archaïques qui ont marqué leur jeunesse :

9 Paul Ricœur, Soi-même comme un autre, Paris, Éditions du Seuil, 1990, p. 148. 10 Émile Miller, Mon voyage autour du monde, Montréal, Bibliothèque de l'Action française, 1923, p. 10. 11 Jacques Hébert et Pierre E. Trudeau, Deux innocents en Chine rouge, Montréal, Les Éditions de l'Homme, 1961, p. 156. 
Ce livre, écrivent-ils, faillit avoir un autre titre : «Le Péril jaune». [...] Nous aurions ainsi rejoint chez plus d'un lecteur l'image que son subconscient conserve de la Chine: pays où grouille une multitude d'hommes jaunes, petits, faméliques, rusés et, plus souvent qu'à leur tour, sinistres. Parmi toutes les frousses au moyen desquelles des éducateurs paranoïaques ont tenté d'effrayer notre enfance - francmaçonnerie, protocole des sages de Sion, bolchevisme, matérialisme américain, botte rouge, Chiniqui et quoi encore? - , le péril jaune avait une place de choix. Jeunes écoliers, nous apprenions par la propagande missionnaire que la Chine était le siège naturel de tous les fléaux: paganismes, pestes, inondations, famines et bêtes féroces; la collecte périodique des timbres de la Sainte-Enfance était aussi une occasion de nous rappeler la condition misérable et quelque peu diabolique d'un peuple qui jetait ses bébés aux pourceaux. Puis les récits d'imagination et d'aventures - mettant en scène les pirates de la mer de Chine et les Fou Man Chou de la pègre changhaïenne [sic] - achevaient de renseigner nos jeunes esprits sur les dangers que recélait l'Empire du Dragon. C'est durant notre adolescence que le péril se précisa. Des professeurs de collège nous démontraient sobrement, chiffres en main, que la poussée démographique ferait bientôt éclater les frontières chinoises et qu'un raz-de-marée jaune aurait tôt fait d'engloutir le monde blanc. Vers cette époque Mr. Believe-it-or-not Ripley répandait aussi une image saisissante: si le peuple chinois défilait quatre par quatre devant un point donné, le défilé - compte tenu des taux de natalité et de mortalité - se poursuivrait pendant toute l'éternité ${ }^{12}$ !

On le voit, les voyageurs déconstruisent une vision archaïque de la Chine, dénoncent ce qu'ils appellent « une ignorance profonde de la mentalité chinoise ${ }^{13}$ ». En fait, comme pour la plupart des voyageurs québécois de la seconde moitié du vingtième siècle, Hébert et Trudeau tentent de contempler l'incertain et l'imprévu sans se barricader derrière des concepts et des constructions préétablis ${ }^{14}$. Alain Grandbois, qui a beaucoup voyagé en Orient pendant l'entre-deux-guerres, fustige également l'attitude condescendante des Occidentaux envers les peuples asiatiques: « [N]ous avons perdu, nous Occidentaux, la partie en Asie par notre maladresse et par notre insolence. [...] Cinq cents millions de Chinois valaient la peine d'un petit effort. Nous ne l'avons pas fait. Nos fils, ou nos neveux, le regretteront peut-être très amèrement un jour» (VM, 251). En 1961, le missionnaire Joseph-Louis Lavoie

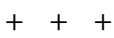

12 Ibid., p. 7-8. 13 Ibid., p. 157. 14 La notion de "péril jaune», qui a hanté le tournant du dix-neuvième siècle, ne considère les Asiatiques que comme des foules menaçantes par leur volume. Au cours de la seconde moitié du vingtième siècle, certains auteurs tentent de se démarquer de cette réduction qui a pour effet de déshumaniser les Chinois, tantôt en relatant leurs rencontres personnelles avec des Asiatiques, tantôt en prenant, de l'intérieur, le contre-pied de ce lieu commun: "Je plonge tout de suite dans une mer de visages jaunes et je suis enchanté, écrit Alain Grandbois. [...] Cette foule de Pékinois qui gesticule mais avec plus de réserve, qui crie, mais sur un mode moins aigu, qui mendie, mais avec plus de discrétion, déjà je l'accepte. » Alain Grandbois, Visages du monde. Images et souvenirs de l'entre-deux-guerres, Montréal, Éditions Hurtubise HMH, 1971, p. 303. Désormais, les références à ce récit seront indiquées par le sigle $V M$, suivi du folio, et placées entre parenthèses dans le texte. 
tente pour sa part d'« exorciser l'un après l'autre un tas de préjugés que les fins-fins de par ici entretiennent contre la Chine ${ }^{15}$ ». Encore à la fin du siècle, nombreux sont les voyageurs qui, comme Hervé Dupuis, avouent avoir à se «déprogrammer ${ }^{16}$ » des « clichés de notre télévision: la prostitution en Thailande, la pauvreté en Inde, la vie tribale en Indonésie » $(V A, 10)$, etc. Dans son récit intitulé Voyage au Levant, Michel Lemieux considère que le voyage doit précisément nous enseigner «à nous décentrer de nos illusions sur notre société et ses couvertures idéologiques» $(V L, 102)$ :

En regardant vivre de près un peuple étranger, de grands pans de notre propre mode de vie se relativisent, au point de devenir suspects. Des manières de penser que l'on croyait «naturelles» acquièrent une distance et se replacent en perspective, laissant apparaître les cordes idéologiques qui les animent. [...] Ces précieuses mises entre parenthèses de sa propre culture transforment forcément le voyageur. $(V L, 101)$

Le récit de voyage retrouve ainsi sa puissance de révélation et devient, un peu comme à la Renaissance avec la découverte du Nouveau Monde, l'occasion d'«interroger le connu à partir de l'inconnu ${ }^{17}$ ", d'adopter une démarche intellectuelle critique à l'égard de la civilisation occidentale. L'éloignement n'est plus alors seulement d'ordre spatial mais aussi culturel, au sens où l'entend Francis Affergan ${ }^{18}$. Le journaliste Jean Pellerin, dont les voyages en Orient sont l'occasion de vivre une expérience de décentrement, semble éprouver une semblable révélation:

Tous ces bains de foule en Asie ont singulièrement modifié mon échelle de valeurs. Contrairement à ce que m'inspirait ma belle assurance d'Occidental, il m'apparut que le monde judéo-chrétien ne tenait pas seul le rôle de dénominateur commun sur notre planète. J'ai même la pénible impression que, loin d'être puissante et vigoureuse, ma civilisation se révèle fragile et minoritaire. Ce déplacement de ce que j'avais cru le centre de gravité de notre monde m'a rendu modeste ${ }^{19}$.

Le récit de voyage en Orient incite donc les auteurs à remettre en question d'anciennes certitudes. À cet égard, certains voyageurs s'intéressent moins au passé mythique de la civilisation orientale qu'à sa situation présente et même à son avenir. Le titre du récit de Jacques de Roussan, Israël, terre de promesses ${ }^{20}$, publié en 1964 , en témoigne. Le premier chapitre de son récit s'intitule « $\mathrm{A}$ la recherche du présent»

$$
+++
$$

15 Joseph-Louis Lavoie, Quand j'étais Chinois, Montréal, Les Éditions Bellarmin, 1961, p. 109.16 Hervé Dupuis, Voir ailleurs. Récit de voyage, Montréal, Triptyque, 1995, p. 83. Désormais, les références à cet ouvrage seront indiquées par le sigle VA, suivi du folio, et placées entre parenthèses dans le texte. 17 Daniel Maggetti, "Voyage», Paul Aron, Denis Saint-Jacques et Alain Viala (dir.), Le dictionnaire du littéraire, Paris, Presses universitaires de France, 2002, p. 635. 18 "La conquête de l'altérité est une aventure déréalisante et qui peut s'avérer dangereuse pour la propre identité de celui qui part. Et ce d'autant plus qu'elle implique une destruction ou pour le moins un abandon du temps et de l'espace identitaires et l'acceptation d'un renversement total des valeurs.» Francis Affergan, Exotisme et altérité. Essai sur les fondements d'une critique de l'anthropologie, Paris, Presses universitaires de France, 1987, p. 44. 19 Jean Pellerin, Escales au bout du monde, Montréal, Guérin , 2000, p. 38-39. 20 Jacques de Roussan, Israël, terre de promesses, [Montréal], Le Cercle du Livre de France, 1964. 
et un autre «Les futurs maîtres d'Israël». Plus encore, chez l'écrivain Jean-Paul Desbiens, les lieux saints perdent leur pouvoir évocateur. Dans son récit intitulé Jérusalem. Terra Dolorosa, publié en 1991, Desbiens s'étonne à quelques reprises du peu d'émotions qu'il ressent en visitant les vestiges du christianisme. Sur le tombeau du Christ, il écrit: "[J]e ne sais quoi dire. Il me semble que je n'avais aucune émotion. Suis-je trop intellectualisé ? Suis-je sec? Ai-je même la foi ${ }^{21}$ ?» Bref, contre toute attente, quelques voyageurs, comme de Roussan et Desbiens, éprouvent peu d'intérêt pour le passé mythique évoqué par les lieux saints, pour l'Orient biblique ou celui des Mille et une nuits. Les relations conflictuelles entre Juifs et Palestiniens, les conséquences de ce conflit dans la vie quotidienne des uns et des autres et ses possibilités de résolutions retiennent davantage leur attention que le mirage de l'Orient hérité de leur culture judéo-chrétienne.

\section{(RE)DÉCOUVRIR L'AUTRE}

Généralement, le décentrement que préconisent les voyageurs à l'égard de leur culture est à la mesure de leur intérêt nouveau pour celle de l'autre. Comme l'ont montré plusieurs études, le vingtième siècle est marqué par un « déplacement radical des modes de perception des sociétés et cultures non occidentales ${ }^{22} »$. L'exotisme, véritable paradigme de perception qui s'est imposé depuis le seizième siècle, pour devenir avec le tourisme du dix-neuvième siècle « un grand voyage-spectacle au sein d'un univers de paysages, monuments, musées ${ }^{23}$ ", cède le pas, à partir des années 1930, à «un changement de perspective au sein duquel les œuvres pionnières de Michel Leiris et Victor Segalen ont servi de relais et de catalyseurs à la prise de parole des Autres et leur "des-exotisation" littéraire et culturelle ${ }^{24}$ ». «Il est incontestable, écrivait Roland Barthes en 1957, que le voyage est devenu (ou redevenu) une voie d'approche humaine et non plus "culturelle" : ce sont de nouveau (peut-être comme au XviII siècle) les mœurs dans leur forme quotidienne qui sont aujourd'hui objet capital du voyage ${ }^{25}$.»

Malgré l'intérêt des voyageurs québécois en Orient pour les sites et les attraits touristiques convenus, leurs récits de voyage témoignent également de cette ouverture à l'autre. Plusieurs auteurs éprouvent de plus en plus le désir de voir, «de rencontrer des gens d'autres pays pour mieux les connaître, mieux les apprécier» (VA, 27), de se mêler, de dire Alain Grandbois, «à tous ces peuples dont les habitudes, les mœurs, le langage, la façon de vivre sont totalement différents» (VM, 371). «Dans toutes mes chroniques, les hommes y occupent une plus grande place que les pierres ${ }^{26}{ }$, écrit le

$$
++
$$

21 Jean-Paul Desbiens, Jérusalem. Terra dolorosa, [Beauport], Éditions du Beffroi, 1991, p. 49.22 HansJürgen Lüsebrink, «La perception de l'autre. Jalons pour une critique littéraire interculturelle», Tangence, n 51 , 1996, p. 63. 23 Edgard Morin, L'esprit du temps, Paris, Grasset, 1983 [1962], p. 82.24 Hans-Jürgen Lüsebrink, loc. cit., p. 63. 25 Roland Barthes, Mythologies, Paris, Éditions du Seuil, 1957, p. 124.26 Eugène Cloutier, Le Canada sans passeport. Regard libre sur un pays en quête de sa réalité, t. II, Montréal, HMH, 1967, p. 293. 
voyageur invétéré Eugène Cloutier, qui publie dans les années 1960 et 1970 des récits de voyage sur le Canada, la Californie, le Japon, la Roumanie, la Suède, la Tunisie, la Turquie, la Yougoslavie, le Chili, l'Allemagne de l'Ouest, le Pays basque et Cuba. «Les monuments, les musées, les sites ne m'intéressent que médiocrement, bien moins en tout cas que les espaces peuplés d'humains, signes vivants ${ }^{27}$ ", constate André Belleau à la fin de son récit au Maroc. Autrement dit, les voyageurs partent à la recherche des nombreux « visages du monde», pour reprendre le titre de l'émission radiophonique animée par Grandbois au début des années 1950. Certains tentent d'acquérir une meilleure connaissance des manières de faire et de vivre dans un espace oriental, voire de s'immerger au milieu des Orientaux, de vivre comme eux. "Nous avons essayé, écrit Jacques Hébert, d'être Japonais au Japon, Indiens aux Indes, Afghans sur les plateaux arides de l'Afghanistan ${ }^{28}$.» Partie à la découverte des différents Visages du Japon, Georgette Lamoureux adopte le mode de vie de ses hôtes au point de se sentir rapidement «japonisée ${ }^{29}$ ». C'est L'Inde vivante ${ }^{30}$, comme l'indique le titre de son récit, qui intéresse Jean-Claude Dussault. À l'instar de plusieurs, il aime errer parmi la foule à la recherche de contacts humains authentiques. De "farang», c'est-à-dire étrangère, qu'elle était au début de son séjour en Thailande, Marie C. Laberge décrit comment elle est parvenue «à gagner l'affection de [son] voisinage, au fil des mois. En vivant comme eux. En [se] fondant dans la foule ${ }^{31} »$.

Ce désir d'immersion des voyageurs marque un important changement d'attitude par rapport à la différence de l'autre. Jusqu'au vingtième siècle, la comparaison des mœurs occidentales et orientales comportait une finalité idéologique, visait essentiellement à jauger les possibilités d'une conversion religieuse. «De l'inversion à la conversion", pour reprendre l'expression d'Affergan, «l'innocence, la nudité, la douceur apparente des mœurs permettent, comme un creux attendant d'être comblé, l'entreprise de la conversion ${ }^{32}$.» Il en résultait une altération de l'altérité, récupérée par la volonté occidentale de transformer le monde à son image, voire à son usage. «En arrachant ces pauvres indigènes à leurs croyances absurdes, notait Léon Provancher en 1884 au sujet des Arabes, on leur inculquera en même temps des idées d'ordre et de travail au moyen desquelles on pourra en faire des citoyens utiles et respectables ${ }^{33}$.» Il en va tout autrement dans la seconde moitié du vingtième siècle. Certes, la figure commode de l'inversion fonctionne toujours comme un principe heuristique dans la mesure où elle permet de comprendre, de donner sens à une altérité qui sans cela resterait complètement opaque ${ }^{34}$, mais elle ne sert plus le processus de conversion:

$$
++
$$

27 André Belleau, loc. cit., p. 55.28 Jacques Hébert, Aventure autour du monde. L'Extrême-Orient en feu, Montréal, Fides, 1952, p. 10. 29 Georgette Lamoureux, Visages du Japon, Sherbrooke, Éditions Paulines, 1969, p. 277. 30 Jean-Claude Dussault, L'Inde vivante. Récit de voyage, Montréal, l'Hexagone, 1990. 31 Marie C. Laberge, En Thailande. Marie au pays des merveilles, Laval, Guy Saint-Jean éditeur, 2003, p. 72. 32 Francis Affergan, op. cit., p. 86.33 Léon Provancher, De Québec à Jérusalem. Journal d'un pèlerinage canadien en Terre-Sainte en passant à travers l'Angleterre, la France, l'Égypte, la Judée, la Samarie, la Galilée, la Syrie et l'Italie, Québec, C. Darveau, 1884, p. 346. 34 "L'inversion est une fiction qui fait "voir" et qui fait comprendre: elle est une des figures concourant à l'élaboration d'une représentation du monde.» François Hartog, Le miroir d'Hérodote. Essai sur la représentation de l'autre, Paris, Éditions Gallimard, 1980, p. 227. 
En Chine, la couleur du deuil est le blanc. Pour signifier un acquiescement, on secoue la tête de droite à gauche. Pour exprimer la négation, le refus, on penche la tête de haut en bas. Autres coutumes! J'ai rencontré certains voyageurs qui se moquaient de ces habitudes, qui ne sont d'ailleurs que des conventions. Mais sontelles plus arbitraires que les nôtres? (VM, 252)

Il n'est pas rare que la comparaison tourne même à l'avantage de l'Orient dont les valeurs sont jugées supérieures à celles de l'Occident. Selon Grandbois, «dans une ville comme celle d'Hanoï, où deux races parfaitement différentes vivent côte à côte, il est très facile, et surtout trop facile, de jouer le jeu des comparaisons. Et ces comparaisons, dois-je l'avouer, ne sont pas toujours, si l'on veut être sincère, à notre avantage » (VM, 262). Chez Jacques Hébert, le renversement des valeurs est marqué avec plus d'acuité encore:

En Amérique, on regarde souvent les Japonais comme de pauvres Jaunes arriérés et sans culture. Or, le raffinement, le sens artistique, les bonnes manières et le confort même que l'on trouve dans une famille bourgeoise japonaise est infiniment supérieur à ce qui existe dans la moyenne des familles bourgeoises du Canada et des États-Unis. [...] Le Canada, pays civilisé qui, à l'occasion, se croit supérieur au Japon, est un pays où les gens ne lisent pas. Un fait notoire. Par contraste, le Japon est un des pays du monde où la population lit le plus ${ }^{35}$.

Les voyageurs en Orient convient donc leurs lecteurs à (re)découvrir l'autre en se délestant d'un savoir ethnocentrique, voire en se «délivr[ant] de nos conceptions habituelles de comparer, de juger»(VM, 339). À cette fin, certains tablent sur cette «irréductibilité éternelle» que préconisait l'écrivain voyageur français Victor Ségalen. Dans son récit de voyage en Tunisie, Eugène Cloutier, par exemple, admet d'entrée de jeu que «parler d'un pays, c'est toujours le trahir un peu». "Quelles que soient vos techniques d'investigation, votre disponibilité d'esprit, votre préparation mentale à vouloir comprendre l'autre et non à le juger, précise Cloutier, vous ne parviendrez jamais qu'à saisir les embruns de sa réalité profonde ${ }^{36}$.» $\mathrm{D}^{\prime}$ autres écrivains voyageurs, notamment Roch Carrier ${ }^{37}$ et Charles Pelletier ${ }^{38}$, tentent d'éviter l'impasse d'un rapport sujet/objet en misant sur la «dépersonnalisation » d'un récit de voyage écrit à la troisième personne du singulier. Ce choix n'est pas sans rappeler le récit de voyage Ecuador d'Henri Michaux, qui, déjà en 1929, employait le «il» comme "une mise à distance, une manière de marquer son doute vis-à-vis de sa propre voix. En présentant la voix narrative comme n'étant pas la sienne, l'auteur se présente comme une non-personne, comme

$$
+++
$$

35 Jacques Hébert, op. cit., p. 99 et 104. 36 Eugène Cloutier, En Tunisie, Montréal, Éditions du Jour, 1970, p. 9. 37 Roch Carrier, Un chameau en Jordanie, [s.l.], Stanké, 1988. Désormais, les références à ce récit seront indiquées par le sigle $C J$, suivi du folio, et placées entre parenthèses dans le texte. 38 Charles Pelletier, Oasis. Itinéraire de Delhi à Bombay, Moncton, Éditions d'Acadie, 1993. Désormais, les références à ce récit seront indiquées par le sigle $O$, suivi du folio, et placées entre parenthèses dans le texte. 
un "je" doutant de lui-même, de sa culture 39 ", souligne Valérie Berty. À tout le moins, ce choix marque une distance évidente par rapport à une tradition qui associait le nom et l'expérience du voyageur occidental à une marque d'authenticité et de crédibilité, et qui pouvait même justifier une forme d'appropriation et de réduction de l'ailleurs, rendu lisible et compréhensible par une sorte de regard supérieur et autorisé.

\section{UNE ALTÉRITÉ INVERSÉE}

Chez plusieurs auteurs, le processus de relativisation des idées reçues se manifeste surtout par une forme d'altérité inversée, ancrée dans un renversement du regard et une prise de parole de l'autre. On sait qu'au dix-neuvième siècle, les voyageurs en Orient se limitaient bien souvent à ne percevoir l'autre que dans sa différence et à lui dénier tout droit de regard et de parole. Or, à partir du vingtième siècle, l'Oriental est de plus en plus doté d'un discours et d'un regard propres qui modifient les conditions de la rencontre. Les voyageurs sont désormais conscients de l'effet qu'ils produisent sur cet autre qui les renvoie à leur propre altérité. «Quand je m'aventure dans les petites rues, dit Jacques Hébert en Corée, je sens peser sur moi un regard qui pourrait être hostile ${ }^{40}$.» À son arrivée à Agra en Inde, Charles Pelletier écrit: «Enfants, adolescents, hommes, vieillards et infirmes s'arrêtent pour le regarder passer. Il fascine comme un numéro de cirque.» $(O, 49)$ Roger Clavet éprouve également «l'impression, après pourtant plus de 20 ans d'ouverture de la Chine au monde extérieur, d'être partout un animal de cirque parce que Iaowai, c'est-à-dire étranger ${ }^{41}$ ». Au Japon, Eugène Cloutier s'étonne d'être davantage pris en photo par les Japonais que l'inverse: "C'est nous qui figurons dans des dizaines d'albums d'écoliers japonais, au titre de curiosité touristique ${ }^{42}$. » Au sujet d'un jeune enfant qui le dévisage, Michel Lemieux écrit: «il est fasciné par quelque chose en moi que je ne saisis pas: je me sens vaguement E.T.!» $(V L, 177)$

Comme l'a bien montré Sarga Moussa, cette impression d'être regardé «trahit un trouble de l'identité chez celui-là même qui devait rester maître du regard». Autrement dit, elle témoigne du «droit de regard sur le regard ${ }^{43}$ », du droit de penser sur la pensée que détient maintenant l'autre:

Ces hommes en robe blanche ont une dignité papale. Le keffieh ajoute un air de mystère. Autre préjugé. Pourquoi un keffieh ajouterait-il au visage plus de mystère qu'une tuque de laine? Lorsqu'un Arabe voit la casquette du Voyageur d'Amérique, pense-t-il que cette coiffure ajoute du mystère à son visage? (CJ, 104; je souligne)

$$
+++
$$

39 Valérie Berty, "Ecuador. Un anti-récit de voyage», Sophie Linon-Chipon, Véronique Magri-Mourgues et Sarga Moussa (dir.), Miroirs de textes. Récits de voyage et intertextualité, Nice, Publications de la Faculté des lettres, arts et sciences humaines de Nice, 1998, p. 134. 40 Jacques Hébert, op. cit., p. 43.41 Roger Clavet, La Chine de ma vie. Un peureux dans l'empire du milieu, Montréal, Stanké, 2001, p. 14.42 Eugène Cloutier, Journées japonaises, Montréal, Éditions du Jour, 1969, p. 21. 43 Sarga Moussa, La relation orientale. Enquête sur la communication dans les récits de voyage en Orient (1811-1861), Paris, Klincksieck, 1995, p. 60. 
Pour Roch Carrier, la véritable rencontre présuppose un point de réversibilité entre le même et l'autre, une découverte réciproque, voire une forme d'empathie sans laquelle la représentation de l'autre n'est pas envisageable, sauf à reproduire les traditionnels poncifs de l'exotisme. Il s'agit en fait de "tenter la contre-épreuve ${ }^{44}$ ", pour reprendre l'expression de Victor Ségalen. Le voyageur ne cherche plus uniquement à décrire l'autre et ce qu'il en a pensé, mais plutôt à révéler ce que sa propre présence a suscité dans le regard et la pensée de l'autre. La réaction qui importe n'est plus uniquement celle du milieu sur le voyageur, mais aussi celle du voyageur sur le milieu. Au sujet de son arrivée à Hiroshima, Eugène Cloutier écrit : «Avec ma caméra, mes lunettes de soleil et mon accoutrement touristique, j'ai eu mal, très mal. “On vient prendre des photos pour la famille... pour montrer ce qu'il en reste". Des tas de pensées du genre devaient remplir la tête, me semblait-il, de tous ceux qui m'apercevaient ${ }^{45}$."

Les paroles et les regards échangés peuvent susciter un malaise chez l'observateur observé et jugé, mais également favoriser une forme de compréhension mutuelle: «Une vieille dame lui demande l'aumône. Il lui donne plusieurs pièces de monnaie. Leurs regards se rencontrent, se fixent... Aucun mot n'est échangé. Plus rien n'existe dans la cité sauf leurs regards.» $(O, 116)$ Cet échange de regards avec un mendiant ou un «itinérant» constitue un topos du récit de voyage en Orient au vingtième siècle. Confrontés à l'étrangeté de l'ailleurs, les voyageurs éprouvent un sentiment de dépossession et de dénuement qui les amène à ressentir une forme de réciprocité dans la rencontre. La réaction de Roger Clavet face à un «miséreux chinois» est typique à cet égard: «[L]'homme m'a regardé droit dans les yeux, sondant jusqu'à mon âme, tout ça, sans prononcer un seul mot, le langage devenant tout à coup inutile ${ }^{46}$.»

L'Oriental acquiert un droit de regard sur le regard, de penser sur la pensée, mais, plus encore, il apprend au voyageur à mieux regarder, à mieux voir: «Fathi, [jeune guide bédouin], écrit Roch Carrier, regarde comme on devrait toujours regarder: avec des yeux neufs. Trop souvent on regarde comme si l'on était aveugle. [...] Je devrais imiter Fathi: regarder cette ville comme on déguste un fruit inconnu.» (CJ, 129-130) À un autre moment, Carrier prend conscience qu'il ne peut apprécier réellement la danse orientale: «Parce qu'il [le voyageur d'Amérique] ne connaissait pas le langage de sa danse, parce qu'il ignorait l'alphabet de ses gestes, il regardait sans voir. Les mouvements de cette danseuse d'Orient avaient été polis par des siècles de tradition. Le Voyageur d'Amérique ne pouvait l'apprécier.» $(C J, 104)$

En fait, de façon générale, l'attitude conquérante, voire condescendante des voyageurs du dix-neuvième siècle, laisse place chez les voyageurs du vingtième siècle à un constat d'ignorance. «Je me suis rendu compte de la profondeur de mon ignorance», écrit Hervé Dupuis (VA, 9). «J'étais un étranger, affirme Roch Carrier, avec mon ignorance, mes préjugés et ma curiosité» $(C J, 8)$. «Nous sommes drôlement ignorants ${ }^{47}$ !»,

44 Victor Ségalen, Essai sur l'exotisme, une esthétique du divers, Fontfroide, Bibliothèque artistique \& littéraire, 1995, p. 17. 45 Eugène Cloutier, Journées japonaises, op. cit., p. 122.46 Roger Clavet, op. cit., p. 190. 47 Ibid., p. 134. 
de dire Roger Clavet. Le voyage au Levant «me montre l'étendue de mon ignorance», constate Michel Lemieux (VL, 175). La langue japonaise, écrit Benoît Lacroix, correspond «à des milliers de syllabes qui font maintenant comme du latin à mes oreilles de plus en plus ignorantes ${ }^{48}$ ». Dans ce contexte contre-ethnocentrique, plusieurs voyageurs admettent leur dépendance à l'égard de l'autre. «Voyager, se dépayser, écrit Jean-Paul Desbiens, c'est se trouver, pour un temps, dans une situation d'ignorance, donc de dépendance ${ }^{49}$.» Plus loin, en traversant le désert, il affirme: "Au désert, on fait l'expérience d'être conduit; l'expérience de la dépendance des autres, de l'Autre ${ }^{50}$.» "Personne n'est plus perdu à l'étranger qu'un étranger précisément ${ }^{51}$ ", de dire Éloi de Grandmont. On est donc loin de la démarche égocentriste des voyageurs du passé, qui réduisaient l'autre à une image dégradée du même. Les voyageurs de la seconde moitié du vingtième siècle témoignent plutôt, au contact de l'autre, de la dégradation de l'image du même:

Qui suis-je, écrit Roch Carrier, pour juger que mon bonhomme [jeune guide] est illettré? N'a-t-il pas appris plusieurs langues entre les falaises de Petra? Qui suisje pour croire que la vie moderne est plus difficile pour lui que pour moi ? Qui suisje pour croire qu'un ordinateur est plus compliqué à dompter qu'un chameau? Moi, pauvre Voyageur d'Amérique diplômé, souvent je me suis découvert illettré, barbare, grossier, dans plusieurs pays du monde. $(C J, 60)$

On le voit, en vertu d'une forme d'altérité inversée, les préjugés anciennement projetés sur l'autre (naïveté, ignorance, barbarie, grossièreté, etc.) se trouvent maintenant attribués au voyageur occidental. On a souvent considéré l'Oriental comme un «enfant», surtout depuis l'avènement de l'industrialisation en Occident qui a accentué, aux yeux des voyageurs, "le caractère "retardé" et "primitif" de l'Orient "arriéré" 52 ». Or, dans les récits de la fin du vingtième siècle en particulier, c'est plutôt l'Occidental qui, malgré son éducation "supérieure», est infantilisé, inadapté, illettré, voire analphabète. À son arrivée à l'aéroport de Narita au Japon, Charles Pelletier se sent comme un enfant qui ne peut lire aucun écriteau, aucune affiche. Même réaction de l'écrivain et membre de l'Académie canadienne-française François Hertel: «[J]e suis complètement illettré: je ne sais ni parler, ni lire, ni écrire ${ }^{53}$.»

Chez certains auteurs, cette inversion est renforcée par la rencontre avec de jeunes enfants orientaux, dont la différence suscite chez le voyageur à la fois la perception d'un manque et le désir de le combler. «J'aimerais parler ton langage comme tu parles le mien» $(C J, 77)$ dit Roch Carrier à son jeune guide Fathi. À Assouan, Louis Valcke rencontre trois jeunes Égyptiennes qui lui font ressentir un

$$
+++
$$

48 Benoît Lacroix, Le Japon entrevu, Montréal, Fides, 1965, p. 19.49 Jean-Paul Desbiens, op. cit., p. 48 50 Ibid., p. 157. 51 Éloi de Grandmont, Voyageurs ou touristes, Montréal, Les Éditions Maisonneuve, 1970, p. 82. 52 Janice Deladalle-Rhodes, «L'altérité dépassée. Doughty et l'Autre», Protée, vol. 22, nº 1, 1994, p. 76 Voir Jean-Claude Berchet, Le voyage en Orient. Anthologie des voyageurs français dans le Levant au XIXe siècle, Paris, Éditions Robert Lafont, 1985. 53 François Hertel, Tout en faisant le tour du monde, Paris, Éditions de la diaspora française, 1971, p. 36. 
sentiment d'infériorité : "Elles me demandent si je comprends l'arabe, paraissent fort déçues de ma réponse négative, et je me sens moi-même quelque peu gêné face à elles et à leur maîtrise de l'anglais ${ }^{54}$.»

Tout se passe comme si voyager exigeait du visiteur une forme de "dépossession, voire qu'il s'abêtit, qu'il redevînt en quelque sorte un enfant ${ }^{55}$ ». Charles Pelletier souligne ce retour nécessaire à l'enfance. Ã ses yeux, son voyage est une «fuite en avant pour mieux saisir l'imaginaire de son enfance» $(O, 21)$. Pour Claude R. Blouin, voyager au Japon constitue « une voie par quoi renouer avec un âge de la vie où, pour la première fois, ce pays vint à notre connaissance ${ }^{56}$ ». "Chaque fois que j'aborde un pays inconnu, écrit pour sa part Jacques Hébert, une singulière émotion m'envahit. Je redeviens un enfant que fascinent le moindre bruit, une odeur nouvelle, une caresse du vent, un geste inédit, un murmure dans le noir ${ }^{57}$ ». Chez Roch Carrier, ce retour aux sensations et aux peurs primitives et instinctuelles de l'enfance traverse tout le récit:

\begin{abstract}
Sous le grand soleil de Jordanie, le Voyageur d'Amérique ressent cet étrange malaise, cette peur atavique, cette inquiétude de se trouver dans une tribu étrangère. Ces gens sont vêtus autrement que lui; leur langue lui est un mystère sonore; leur peau n'a pas la pâleur de la sienne, l'odeur de leur corps ne lui est pas familière ; il ressent un certain inconfort, peut-être une peur comme l'enfant peut avoir peur de la nuit. Cette crainte est une réaction devant l'inconnu. Il voyage pour ressentir le plaisir de ce malaise. (CJ, 131-132)
\end{abstract}

Plus qu'à une régression au monde de l'enfance, les voyageurs et les lecteurs sont conviés à vivre une mort et une renaissance symboliques, à mourir à leur occidentalité pour renaître en développant certaines qualités essentielles: une "écoute sensible»; l'humilité; la modestie; l'empathie. Pour certains, il s'agit d'un véritable appel à la métamorphose. Charles Pelletier, par exemple, dépouillé de son «je» dans son récit de voyage écrit à la troisième personne du singulier, pousse plus loin le rêve nervalien d'être autre, en tentant de camoufler tous les signes extérieurs de son identité occidentale :

Il choisit de se lancer à corps perdu dans sa passion, comme une dérive. De s'imbriquer dans ce corps à corps qu'est la réalité indienne. Se fondre dans cette humanité. Depuis toujours il aime les bains de foule, mais ils prennent en Inde des dimensions insoupçonnées. L'humanité a ses couleurs. Il doit apprendre à les percevoir comme elles sont. Avant de quitter sa chambre ce matin-là, il se dépouille de tout ce qui le rattache ou l'identifie à cet ailleurs (l'Amérique) et des objets de désir: jeans, chemises, T-shirts... Il ne conserve que quelques vêtements qu'il a

$$
++
$$

54 Louis Valcke, Vignettes égyptiennes, Sherbrooke, Éditions G.G.C., 2003, p. 167. 55 Serge Cantin, Nous revoilà rendus au sol. Essais sur le désenchantement du monde, Montréal, Bellarmin, 2003, p. 28.56 Claude R. Blouin, Du Japon et d'ici, Montréal, Éditions Pleins bords, 1975, p. 11.57 Jacques Hébert, Yémen. Invitation au voyage en Arabie heureuse, Montréal, Éditions Héritage, 1989, p. 11. 
achetés depuis son arrivée. [...] C'est le même matin qu'il décide de laisser pousser sa moustache, à la manière des hommes de l'Inde. Anonymat. Il adopte de nouveaux stéréotypes et modifie son «langage». Il est appelé à jouer le plus grand rôle de sa vie, devenir un Indien. Métamorphose, incarnation et, par analogie, Vishnou. La vie est une scène où se jouent les vicissitudes de nos existences. $(0,76-77)$

L'expérience de l'altérité éveille chez le voyageur le désir fantasmatique d'adopter une nouvelle identité, de changer de personnalité, de s'ouvrir à «soi-même comme un autre», pour reprendre le titre d'un ouvrage de Paul Ricœur ${ }^{58}$. Ainsi que le constate Michel Lemieux dans son Voyage au Levant, un séjour à l'étranger met en branle une «mécanique imprévue de dénuement social » $(V L, 203)$, un processus de dilution et de dépersonnalisation:

À la longue, perdus dans des villes si différentes, soumis à des climats dépaysants et aux agitations puissantes de ces foules qui nous ignorent, de grands pans de notre image personnelle se diluent peu à peu. [...] Dans ce tourbillon de gens, de langues et de kilomètres, je ne ressens plus ma place sociale, je deviens une planète anomique libérée de sa galaxie et qui erre sans gravité sociale, un personnage neutre qui n'appartient plus à un métier, à une classe sociale, à une région ou à une famille. Je flotte dans l'indistinct social, ayant brisé mes attaches ordinaires. (VL, 203)

On assiste donc à une modification importante de l'horizon autobiographique auquel la pratique du récit de voyage en Orient a souvent été associée depuis le dixneuvième siècle ${ }^{59}$. En effet, l'approche chateaubrianesque, qui a servi de modèle à des générations d'écrivains voyageurs, se limitait en grande partie à la quête des origines, à «ce stade du miroir dont la fonction est sans doute de permettre au sujet infans de trouver l'image réunifiée de lui-même grâce à laquelle il pourra dépasser le sentiment de son morcellement ${ }^{60} »$. Or, dans les récits de la seconde moitié du vingtième siècle, se retrouver importe moins que de se perdre, car l'image réunifiée de soi exige préalablement la perte de soi et de ses repères, la «désinsertion de soi dans le cadre d'une expérience de socialisation culturellement excentrée ${ }^{61}$ ». L'écrivain suisse Nicolas Bouvier propose au sujet de cette difficile métempsycose une image particulièrement évocatrice :

Combien d'années encore pour avoir tout à fait raison de ce moi qui fait obstacle à tout? Ulysse ne croyait pas si bien dire quand il mettait les mains en cornet pour hurler au Cyclope qu'il s'appelait «Personne». On ne voyage pas pour se garnir

$$
+++
$$

58 Paul Ricœur, op. cit. 59 Voir Jean-Claude Berchet, «Un voyage vers soi», Poétique, n 53, 1983, p. 91108; Pierre Rajotte, "Le récit de voyage au XIXe. Une pratique de l'intime», Globe. Revue internationale d'études québécoises, vol. 3, nº 1, 2000, p. 15-37. 60 John E. Jackson, Passions du sujet, Paris, Éditions Mercure de France, 1990, p. 111. 61 Jean Didier Urbain, «Entretien», Franck Michel (dir.), Tourismes, touristes, sociétés, Paris, Éditions L'Harmattan, 1998, p. 364. 
d'exotisme et d'anecdotes comme un sapin de Noël, mais pour que la route vous plume, vous rince, vous essore, vous rende comme ces serviettes élimées par les lessives qu'on vous tend avec un éclat de savon dans les bordels. On s'en va loin des alibis ou des malédictions natales, et dans chaque ballot crasseux coltiné dans des salles d'attente archibondées, sur de petits quais de gare atterrants de chaleur et de misère, ce qu'on voit passer c'est son propre cercueil. Sans ce détachement et cette transparence, comment espérer faire voir ce qu'on a vu ${ }^{62}$ ?

La différence de l'ailleurs et de l'autre incite donc le voyageur à se dépouiller de ses appartenances sociétales, à larguer ses amarres et ses attaches culturelles souvent perçues comme un enchaînement. "Le temps d'une cavale, j'ai sauté le mur, écrit Michel Lemieux. [...] Me voilà socialement vierge, suspendu hors société; je n'ai plus ni âge ni attaches» (VL, 205). «Je me suis aussi aperçue combien cet exil dans le monde asiatique avait changé ma façon d'être et d'agir ${ }^{63}$ ", estime pour sa part Marie C. Laberge. Autant dire que plus ils avancent, plus les voyageurs se délestent, se décentrent, se vident, se transforment. Cette déperdition temporaire de soi, cette «nouvelle forme de présence à soi ${ }^{64}$ » constitue une véritable quête de sens et, bien souvent, le but de leur expérience viatique.

\section{CONCLUSION}

On constate, aux plans tant des motivations que des méthodes, qu'un certain nombre de tendances viennent renouveler la représentation de l'Orient dans les récits des voyageurs québécois. Pendant tout le dix-neuvième siècle et le début du vingtième siècle, l'Orient ne présente d'intérêt que dans la mesure où il permet aux voyageurs de réactualiser les mythes et les images archaïques. C'est donc l'Orient que l'on connaît que l'on recherche, c'est-à-dire l'Orient de la Terre sainte, d'un passé mythique, l'Orient conforme à des préjugés favorables ou défavorables. Dans ces conditions, de dire Véronique Magri, "le discours sur l'Autre se réduit à un discours du même au même sur l'Autre qu'on essaie de ramener encore au Même ${ }^{65}$ ».

Il en va tout autrement dans la seconde moitié du vingtième siècle, alors que la quête du connu laisse place à celle de l'inconnu. «On se déplace pour être surpris et étonné ${ }^{66}$ ", affirment Louise Blanchard et André Dalcourt dans leur récit de voyage Sabbatique asiatique. Plutôt que la Terre sainte, les écrivains voyageurs préfèrent les régions moins fréquentées de l'Orient et de l'Extrême-Orient. Le présent et l'avenir des peuples orientaux se substituent peu à peu à l'évocation

$$
++
$$

62 Nicolas Bouvier, Euvres, Paris, Éditions Gallimard, coll. "Quarto», 2004, p. 748.63 Marie C. Laberge, op. cit., p. 77. 64 Éric Landowski, Présence de l'autre. Essai de socio-sémiotique, t. II, Paris, Presses universitaires de France, 1997, p. 99. 65 Véronique Magri, Le discours sur l'autre à travers quatre récits de voyage en Orient, Paris, Champion, 1995, p. 405.66 Louise Blanchard et André Dalcourt, Sabbatique asiatique, t. II. Récit de voyage, Outremont, Lanctôt Éditeur, 2000, p. 78. 
exclusive de leur passé mythique. Le désir de retrouver sa culture dans celle de l'autre laisse place à la volonté de se désaliéner d'une perception ethnocentrée au profit d'une meilleure (re)connaissance de l'autre et d'une réelle connivence avec le milieu. Chez certains auteurs, cette nouvelle lecture du monde conduit à une tentative de dédoublement pouvant aller jusqu'à l'inversion des rôles: l'autre fait l'objet d'une idéalisation exotisante et les préjugés séculaires qui lui étaient anciennement attribués caractérisent maintenant le voyageur occidental. À la limite, le discours de ce dernier «n'a le plus souvent pour fonction que de constituer l'autre en modèle de bonté originelle et naturelle, de s'accuser et de s'humilier, d'exhiber son être-inacceptable dans un miroir contre-ethnocentrique ${ }^{67}$ ", pour reprendre les mots de Jacques Derrida au sujet des Tristes tropiques de Claude Lévi-Strauss.

À la fin du vingtième siècle, toutefois, cette volonté de "décomprendre le monde» $(M I, 116)$ mène plutôt une interrogation ouverte à la fois sur l'attribution et sur l'insuffisance du sens inhérentes à nos rencontres avec la différence de l'ailleurs et de l'autre. Pour les écrivains voyageurs, le défi consiste alors à sortir du dilemme séculaire entre la négation de l'autre et l'idéalisation de ce même autre. Ces deux approches diamétralement opposées ont "en commun de semblables stratégies d'évitement psychologiques ("Vermeidungsstrategien") détournées de toute tentative sérieuse de compréhension et de connaissance de l'Autre ${ }^{68} »$. En outre, comme le mentionne Tzvetan Todorov au sujet de l'expérience exotique de Victor Ségalen, «pour éprouver l'autre, on n'a pas besoin de cesser d'être soi ${ }^{69}$ ». Dans son récit de voyage au Tibet (2002), André Carpentier considère, à l'instar de Ségalen, que prendre la mesure du Divers ne doit pas consister à tenter illusoirement de s'identifier à l'autre, ni se prendre pour lui ni le prendre pour soi. Il s'agit plutôt «de séjourner dans la pensée de l'altérité sans espoir de vérité» $(M I, 116)$, «de réapprendre à tourner le dos à son monde, de manière à entrer, non pas seulement dans le monde de l'autre, ce qui ne serait pas si difficile, mais, dirait-on, cette fois-ci, dans un monde autre, où nos croyances et nos valeurs seront hors norme» (MI, 29). Jocelyn Létourneau parle pour sa part de «migrance contrôlée ou mesurée vers l'autre et l'ailleurs", dont le but, pour de plus en plus de Québécois, est

d'incorporer, au stock de références par rapport auxquelles ils se définissent positivement et fièrement, de nouvelles références qui modifieront partiellement, mais non pas radicalement ou totalement, la configuration culturelle qui les caractérise. On pourrait conceptualiser cette démarche d'ouverture à l'autre et à l'ailleurs par le terme (emprunté à J.-C. Charles) d'enracinerrance, soit cette volonté d'aller vers l'autre et vers l'ailleurs, mais à partir de soi et pour y revenir ${ }^{70}$.

$$
+++
$$

67 Jacques Derrida, De la grammatologie, Paris, Les Éditions de Minuit, 1967, p. 168.68 Hans-Jürgen Lüsebrink, loc. cit., p. 53. 69 Tzvetan Todorov, Nous et les autres. La réflexion française sur la diversité humaine, Paris, Éditions du Seuil, 1989, p. 367.70 Jocelyn Létourneau, «L'altérité chantée, l'altérité vécue. Conceptualiser l'échange culturel dans le Québec contemporain ", Pierre Ouellet (dir.), Le soi et l'autre. L'énonciation de l'identité dans les contextes interculturels, Sainte-Foy, Les Presses de l'Université Laval, 2003, p. 441. 
Il y a tout lieu de croire que c'est là la quête que poursuivra le voyageur du vingtunième siècle, qui est de plus en plus conscient qu' « on ne peut partir qu'avec soimême et ce que l'on est, sans feindre une altérité qui ne serait toujours que factice » $(M I, 46)$. Après le même, puis l'autre, l'écrivain voyageur part désormais à la recherche d'un soi plus ouvert à la diversité et à la part de sa propre identité que lui confère l'autre. À la fin de son voyage en Asie, Hervé Dupuis résume bien les résultats de cette approche:

Je suis allé là-bas ni pour leur parler d'un nouveau dieu, ni pour leur imposer ma culture [...]. Je n'allais pas là-bas non plus pour devenir comme eux. Je n'ai adopté ni leur culture ni leur religion. Je reviens avec les mêmes habits, les mêmes habitudes, ma personnalité. Mais j'aurai changé un peu. Je ne peux plus voir le monde de la même façon. Ils m'ont appris qu'il existait d'autres modes de vie, d'autres visions du monde, fort différents des miens, dont je devrai dorénavant tenir compte. (VA, 210)

Entre leur culture et celle de l'autre, les écrivains voyageurs d'aujourd'hui reconnaissent de plus en plus que persistera toujours une certaine différence qui ne doit pas être considérée nécessairement comme une entrave à la réciprocité, mais plutôt comme une incitation à poursuivre perpétuellement le dialogue et l'échange. En ce sens, leur quête correspond plus que jamais à «l'Esthétique du Divers» telle que la proposait Victor Ségalen, c'est-à-dire qu'elle ne vise plus à atteindre «la compréhension parfaite d'un hors soi-même qu'on étreindrait en soi, mais la perception aiguë et immédiate d'une incompréhensibilité éternelle ${ }^{71} »$.

71 Victor Ségalen, op. cit., p. 25. 